\title{
GESTIÓN INTEGRADA DE LA SUBCUENCA DEL ARROYO LEÓN - HONDO EN EL OCCIDENTE DEL SUELO URBANO DEL DISTRITO DE BARRANQUILLA, COLOMBIA
}

\section{INTEGRATED MANAGEMENT OF ARROYO ARROYO LEÓN - HONDO SUBBASIN IN THE WEST URBAN LAND DISTRICT OF BARRANQUILLA, COLOMBIA}

FECHA DE RECIBO: OCTUBRE 31, 2012

FECHA DE ACEPTACIÓN: DICIEMBRE 20, 2012

\section{FREDDY A. SANTIAGO MOLINA}

Arquitecto (Pontificia Universidad Javeriana, 1978), Especialista en Planificación Regional. (CIDER - Universidad de los Andes \& Instituto de Estudios Sociales de La Haya, 1979), Magister en Planificación y Administración del Desarrollo Regional (Universidad de los Andes, 1984), Diplomado en Planeación de Espacios de Trabajo para la Prevención de Riesgos (Universidad del Atlántico, 2003), Diplomado en Definición de Políticas del Suelo en Pequeñas Ciudades (Lincoln Institute of Land Policy - EAD, 2010) y Especialista en Gestión Ambiental Urbana (Universidad Nacional del Centro del Perú - ONG Fondo Verde, 2011). Profesor del Programa de Arquitectura de la Universidad Autónoma del Caribe. Grupo de investigación “

Arquitectura Bioclimática". santiagomolinna@yahoo.com

\section{RESUMEN:}

Este artículo de muestra los resultados de una investigación que concluyó en una Propuesta de Plan de Gestión Integral de la Subcuenca del Arroyo León - Hondo, la cual se formuló con el enfoque de Gestión Integrada de Cuencas y dentro del concepto de gestión del riesgo, entendida esta como el conjunto de herramientas tendientes a reducir la vulnerabilidad de un territorio frente a amenazas del entorno y para reducir su riesgo ambiental. La formulación del Plan de Gestión Integral de la Subcuenca del Arroyo León - Hondo se enmarca en el enfoque metodológico de Fuerzas Motrices - Presión - Estado - Impacto - Respuestas (FMPEIR), propuesto por el PNUMA, el cual analiza las interrelaciones entre la sociedad humana y el medio ambiente haciendo énfasis en los servicios de los ecosistemas y su relación con el bienestar humano.

\section{PALABRAS CLAVE:}

Gestión integrada de cuencas hídricas, riesgo, gestión del riesgo, fuerzas motrices, presiones, estado, impacto, respuestas, cuencas hídricas.

\section{ABSTRACT:}

This article shows concluded research results of a Comprehensive Management Plan of the Arroyo Leon - Hondo Sub-basin proposal formulated from the approach of Integrated Watershed and within the risk management concept, understood as a set of tools aimed at reducing the vulnerability of a territory against environmental threats and reduces their environmental risk. The formulation of the Comprehensive Management Plan of the Arroyo Leon -Hondo Sub-basin is part of Driving Forces - Pressure - State - Impact - Responses, DFPSIR, a methodological approach proposed by UNEP, which analyzes the interrelationships between human society and environment with an emphasis on ecosystem services and their relationship to human welfare.

\section{KEYWORDS:}

Integrated watershed, risk, risk management, driving forces, pressure, state, impact, responses, watershed management 
... pareciera que la naturaleza tuviera dos caras, como el antiguo dios romano Jano:

La cara sonriente, a la que hay que proteger y la cara amenazante, contra la que hay que protegerse, ...pero las dos son caras del mismo ente, y los ritos realizados ante cualquiera de los dos rostros tendrán consecuencias sobre el otro ...

Michel Hermelin (1993) ${ }^{1}$

o es revelar un secreto decir que el agua es esencial para la vida, no solo por su condición bioquímica para el consumo humano, sino porque sostiene actividades productivas (agricultura, industrias, pesca, turismo, etc.)

En el otro extremo, el agua fuera de control, sin manejo, volcada sobre el territorio, puede convertirse en fuente de destrucción y muerte: acabar con la riqueza, eliminar vidas, transmitir enfermedades o inundar extensas áreas. Un déficit en la cantidad o en la calidad de agua disponible puede provocar el deterioro en la salud humana (y hasta la muerte) y producir un serio deterioro económico.

Entre estos dos extremos, la competencia por el uso del agua puede generar $\mathrm{y} / \mathrm{o}$ intensificar conflictos entre las comunidades en una cuenca local, nacional o internacional cuando se trata de cuencas transfronterizas compartidas por más de un país.

Los mencionados conflictos están asociados a la diversidad de presiones a que están sometidos los recursos hídricos, las cuales evidencian las interdependencias territoriales, hidrológicas, sociales, económicas y ecológicas que existen en las cuencas hidrográficas, lacustres y acuíferas, no importa el tamaño o la superficie que abarquen las mismas: desde la microcuenca de un arroyo o riachuelo hasta la macrocuenca que define una región natural nacional, en tanto constituyen el marco territorial de los recursos hídricos. Estas interdependencias exigen enfoques más integrados para el desarrollo y la gestión de los recursos del agua y de la tierra.

Dentro de los límites de una cuenca, integrar los usos del suelo y la gestión del agua es una tarea que implica entender que la gestión del suelo (que incluye la planificación del ordenamiento de sus usos e intensidades de ocupación, la silvicultura, la industria, la agricultura y el medio ambiente) generalmente está regulada por políticas no relacionadas con las normas del agua y está administrada por sectores de diversas características en una administración y utilizada por actores no siempre con intereses comunes.

La cantidad y calidad del agua en los ecosistemas es modificada por las diversas formas en que la sociedad utiliza el agua, la contamina o le modifica la morfología de sus cursos, cauces

o cuerpos de agua, alterando su valor intrínseco y afectando sus servicios ambientales, esenciales y de enorme valor para el bienestar humano.

La naturaleza multidimensional de la gestión del agua asociada a su territorio es lo que aquí se entiende como la gestión integrada de recursos hídricos (GIRH), con la especificidad que le determina la delimitación territorial de su cuenca.

\section{" La importancia del concepto gestión del riesgo radica en que permite identificar cuáles dinámicas de la naturaleza se consti- tuyen en amenazas contra las comunidades humanas y cuáles dinámicas sociales se constituyen en amenazas contra los eco- sistemas, al igual de identificar los factores que hacen vulnerables a dichas comuni- dades y ecosistemas. "}

Bajo este enfoque, la GIRH con enfoque de cuencas, ayuda a administrar y desarrollar cualquier recurso hídrico en forma sostenible y equilibrada, teniendo en cuenta la diversidad de intereses sociales, económicos y ambientales de los diversos agentes que actúan en la respectiva cuenca; reconociendo los diferentes grupos de interés que compiten entre sí por los recursos de la cuenca; ordenando los diversos sectores que usan y abusan del agua; y dimensionando las necesidades de ordenamiento y planificación ambiental y territorial de la misma.

En este contexto, es referente fundamental la proposición de la GWP 2(2000), según la cual la Gestión Integrada de Recursos Hídricos en cuencas (GIRH) se define como un proceso que promueve el desarrollo y gestión coordinados del agua, la tierra y los recursos asociados, para maximizar el resultante bienestar económico y social de manera equitativa sin comprometer la sostenibilidad de ecosistemas vitales. (p. 18)

Bajo el enfoque enunciado, se desarrolló la investigación que condujo a una propuesta de Gestión integrada de la Subcuenca del Arroyo León - Hondo en el occidente del suelo urbano del Distrito de Barranquilla (Colombia), en el cual definieron elementos fundamentales -territoriales, sectoriales y de los grupos de interés- para la gestión integral del territorio 
ARTE \& DISEÑO, ISSN: 1692-8555, Vol. $11 \mathrm{~N}^{\circ} 1$, Enero - Junio 2013 Gestión integrada de la subcuenca del arroyo León - Hondo en el occidente del suelo urbano del distrito de Barranquilla, Colombia, págs. 47-62

asociado al Arroyo León-Hondo, cauce de origen pluvial, la cuenca más importante del occidente y noroccidente de Barranquilla, la ciudad más grande e importante de la Región Caribe de Colombia.

En dirección a lo anterior, la gestión integrada de la Subcuenca del Arroyo León - Hondo analiza las diferentes escalas de ocupación de la cuenca y su interacción con escalas territoriales superiores; define criterios para lograr una adecuada gobernabilidad sobre la cuenca; y propone algunos mecanismos para instituir acuerdos ${ }^{3}$ normativos e institucionales efectivos que permitan tomar decisiones ambientalmente sustentables, socialmente equitativas y favorables para el crecimiento económico de la cuenca; finalmente, plantea instrumentos que serían extrapolables a otros ámbitos territoriales más amplios y complejos.

Sobre la subcuenca del Arroyo León - Hondo gravitan, con mucho peso, fenómenos resultantes de la dinámica urbana de Barranquilla, tales como: inestabilidad de suelos en las laderas que le bordean; contaminación por residuos líquidos y sólidos de origen industrial, comercial y doméstico; conflicto en los usos del suelo; y ocupación irregular por la expansión de actividades urbanas, entre otros. Estos problemas están asociados principalmente a las presiones ambientales a que está sometida: deforestación, sedimentación de cauces, vertimientos de aguas residuales sin tratar, deposición de basuras de toda clase, invasión de rondas de los cuerpos de agua y ausencia de una cultura socio-ambiental; todo ello enmarcado por un contexto institucional caracterizado por la duplicidad y/o traslapo de funciones, débil capacidad de control de las entidades responsables del ordenamiento ambiental y/o territorial en el ámbito de la cuenca, produciendo en ella impactos como: elevados niveles de contaminación de los suelos y aguas; pérdida de la capacidad hidráulica de los arroyos; ausencia de una clara ordenación del territorio; inexistencia de criterios para la gestión de riesgos; y una baja gobernabilidad sobre la cuenca.

En dirección al anterior marco problemático, la gestión integrada de la Subcuenca del arroyo León - Hondo en el occidente del suelo urbano del Distrito de Barranquilla (Colombia) se enmarca teóricamente en el concepto de la gestión del riesgo, entendida ésta, según Ornés \& Chacón (2009) como el conjunto de medidas y herramientas tendientes a reducir la vulnerabilidad de un territorio frente a amenazas del entorno y para reducir su riesgo ambiental (p. 1).

La importancia del concepto gestión del riesgo radica en que permite identificar cuáles dinámicas de la naturaleza se constituyen en amenazas contra las comunidades humanas y cuáles dinámicas sociales se constituyen en amenazas contra los ecosistemas, al igual de identificar los factores que hacen vulnerables a dichas comunidades y ecosistemas.
Bajo esta óptica, la gestión integrada de la Subcuenca del Arroyo León - Hondo, bajo el enfoque metodológico desarrollado por el PNUMA (2010), denominado FMPEIR (Fuerzas Motrices - Presión - Estado - Impacto - Respuestas), cubre y analiza las interrelaciones entre la sociedad humana y el medio ambiente haciendo énfasis en los servicios de los ecosistemas y su relación con el bienestar humano.

\section{" La gestión integrada de la Subcuenca del Arroyo León - Hondo, bajo el enfoque meto- dológico desarrollado por el PNUMA (2010), denominado FMPElR (Fuerzas Motrices - Presión - Estado - Impacto - Respuestas), cubre y analiza las interrelaciones entre la sociedad humana y el medio ambiente haciendo énfasis en los servicios de los ecosistemas y su relación con el bienestar humano."}

Por ello, es referente fundamental según la cual la Gestión Integrada de Recursos Hídricos -GIRH- en cuencas se definiría como un proceso que promueve el desarrollo y gestión coordinados del agua, la tierra y los recursos asociados, para maximizar el resultante bienestar económico y social de manera equitativa sin comprometer la sostenibilidad de ecosistemas vitales. (GWP \& INB0, 2009, p. 18)

A la luz de lo anterior, una propuesta de gestión integral de la Subcuenca del Arroyo León - Hondo impactaría favorablemente sobre: la gobernabilidad de la cuenca, por cuanto se propone formular un nuevo esquema de coordinación interinstitucional que haga eficaces las acciones sobre éstas; la sustentabilidad de las estructuras ambientales, por cuanto las propuestas se enfocarán bajo el concepto de la gestión integral del riesgo; el ordenamiento ambiental y territorial, en tanto su administración y control se propondrán en el marco de un modelo de uso e intensidad de ocupación del territorio que contemple, dentro de sus objetivos estratégicos, la minimización de la vulnerabilidad y de la susceptibilidad existente en la cuenca.

\section{MARCO GENERAL DE LA INVESTIGACIÓN Y OBJETIVOS DEL PLAN}

El marco problemático de la investigación es un territorio caracterizado por la multiplicidad de ámbitos determinados por 
las diversidad de instituciones de distinto nivel que tienen jurisdicción sobre él y que limitan la capacidad de respuesta; por el fuerte impacto que sobre él ejercen las fuerzas motrices de la dinámica urbana; por la existencia de un entorno que combina lo rural, los relictos naturales y una fuerte actividad urbana; y por una baja gobernabilidad y control institucional sobre el territorio.

En el anterior contexto, la gestión integrada para la Subcuenca del Arroyo León - Hondo propone un esquema gestión territorial y ambiental que propugne por la búsqueda de un desarrollo sostenible, equilibrado y ordenado del territorio asociado al recurso hídrico del área objetivo, por medio de la formulación de un instrumental analítico que conduzca la estructuración de mecanismos operativos y de coordinación interinstitucional que permitan hacer operativas y "materializables" un conjunto de respuestas al deterioro ambiental y territorial que soporta la microcuenca. Así entonces, se propusieron los siguientes objetivos específicos:

- Contribuir a la reducción de los factores de riesgo ambiental 50 beneficios de los demás, por lo cual es necesario buscar "un balance razonable" (p. 53).

Desde 1992, tanto en la Conferencia Internacional sobre el Agua y el Medio Ambiente (Dublín, Irlanda, enero de 1992) - El Desarrollo en la Perspectiva del Siglo XXI- como en la Conferencia de las Naciones Unidas sobre el Medio Ambiente y el Desarrollo (Río de Janeiro, junio de 1992) -Cumbre de la Tierra-, se reconoce consensualmente por los países del orbe que la entidad geográfica más apropiada para la planificación y gestión de los recursos hídricos es la cuenca fluvial" y que la "ordenación integrada de los recursos hídricos se basa en la percepción de que el agua es parte integrante del ecosistema, un recurso natural y un bien social y bien económico (Solanes y Jouravlev, 2005, p. 52).

\section{"La investigación asume la interpretación de Solanes y Jouravlev (2005), la teoría de la gestión integrada del recurso hídrico reconoce sus fundamentos en los valores del sistema, según los cuales, en un mismo territorio (la cuenca) no pueden maximi- zarse simultáneamente los beneficios para todos los usos del agua. Lo anterior significa que no se pueden maximizar los beneficios para un usuario 0 actor sin afectar negativamente los beneficios de los demás, por lo cual es necesario buscar "un balance razonable" (p. 53)."}

Luego de las anteriores afirmaciones, los países reunidos en las conferencias de las Naciones Unidas concluyen que dicha gestión, "incluida la integración de los aspectos relativos a las tierras y a las aguas, tendría que hacerse a nivel de cuenca 0 subcuenca de captación". (Solanes y Jouravlev, 2005. p. 52)

Finalmente, la definición del territorio de la cuenca como unidad de comprensión para la gestión integral de sus recursos se contextualiza en diversos marcos teóricos (adaptado de Solanes y Jouravlev, 2005, p.41), a saber:

- Desde las teorías físicas e hidráulicas, porque las cuencas deben ser entendidas como las principales formas te-rrestres dentro del ciclo hidrológico que captan y concentran la oferta del agua que proviene de las precipitaciones. 
ARTE \& DISEÑO, ISSN: 1692-8555, Vol. $11 \mathrm{~N}^{\circ} 1$, Enero - Junio 2013

- Desde la óptica de las teorías del desarrollo y ordenamiento del territorio, porque las interrelaciones existentes entre usos y usuarios de la cuenca la convierten en la unidad territorial adecuada de análisis para la toma de decisiones de gestión del agua, especialmente en cuanto a su uso múltiple, su asignación y el control de su contaminación.

- Desde las teorías ambientales y biofísicas, porque el ámbito de la cuenca se erige en el sistema territorial en el que se producen los procesos permanentes y dinámicos de interdependencia e interacción del agua con los sistemas físico (recursos naturales) y biótico (flora y fauna).

- Desde las teorías socioeconómicas, porque en las cuencas se produce la interrelación e interdependencia entre los sistemas físicos y bióticos, y el sistema socioeconómico, formado por los usuarios de las cuencas, sean habitantes o interventores externos de la misma.

De otra parte, para entender el marco teórico de la gestión del riesgo se tiene como punto de partida los conceptos asociados al riesgo, en dirección a lo cual se proponen varios referentes que permitirán tener un panorama conceptual amplio y diverso, que eventualmente también conduzca a nuevas interpretaciones en un tema aún en elaboración por parte de diversas disciplinas.

Jorge Olcina Cantos (2008) hace una disquisición epistemológica alrededor de la etimología y el origen del término riesgo (pp. 4 - 9), recordando las definiciones del Diccionario de la Real Academia (hasta su $22^{\mathrm{a}}$ edición): resgar (cortar), proce-

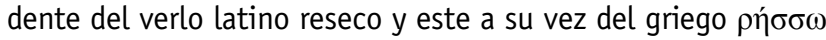
(romper, desgarrar), haciendo alusión a la idea de ruptura de la normalidad que supone la asunción de un riesgo; luego recuerda que riesgo procede del italiano risico ó rischio, expresión que deriva del árabe clásico rizq, que significa lo que depara la providencia.

Despojando a este último significado de su connotación sobrenatural, afirma Olcina Cantos (2008) que de un modo u otro, subyace la idea de que el riesgo supone el desarrollo de una nueva situación y, generalmente, una ruptura con lo anterior que abre un nuevo escenario de incertidumbre; y continúa su elaboración citando a Nocenzi (2002), quien relaciona en concepto riesgo con la innata propensión que desde la Antigüedad, ha tenido el ser humano a indagar en lo desconocido, a pesar de las consecuencias negativas que ello podía suponer; mencionando luego a Sennett (1998), según el cual la palabra riesgo (risicare) se encontraría en la obra Liber Abaci (1202) de Leonardo Fibonacci «El Pisano»; para continuar 4 sus anotaciones etimológicas con una referencia al sociólogo inglés Anthony Giddens (2003) quien encuentra el origen del concepto riesgo en la edad Moderna, en el empleo que de esta

Citando la obra Un mundo desbocado. Madrid: Ed. Taurus. palabra realizaron los exploradores occidentales en sus viajes por el mundo y según el cual, la expresión habría llegado al inglés a través del español o del portugués, donde se usaba para referirse a navegar en aguas desconocidas, [de manera que] el concepto estuvo, en sus orígenes, vinculado al espacio geográfico. Concluye Olcina Cantos (2008) anotando que la primera edición de la Enciclopedia Británica (1771) se refiere al término risk vinculándolo con la probabilidad en el juego y que en la actualidad, se emplea para referirse a una amplia gana de situaciones de incertidumbre (p. 9).

Finalmente, el mismo Olcina Cantos (2008), para resaltar la diferencia existente entre riesgo natural y desastre natural, se remite a Aristóteles quien propone la dicotomía entre la potencia y el acto en tanto la interpretación del movimiento en cuanto devenir, según la cual debe entenderse que riesgo y catástrofe se asimilan a los términos de posibilidad y realidad y recuerda que los anglosajones hablan de «natural risk» y «natural hazard», para diferenciar los conceptos de riesgo y catástrofe, respectivamente.

Por su parte, la ONU (2004) define el riesgo como la probabilidad de consecuencias perjudiciales ó pérdidas esperadas (muertes, lesiones, propiedad, medios de subsistencia, interrupción de actividad económica o deterioro del ambiente), resultado de interacciones entre amenazas naturales o antropogénicas y condiciones de vulnerabilidad. Convencionalmente el riesgo es expresado como función de amenaza, vulnerabilidad y capacidad. Algunas disciplinas también incluyen el concepto de exposición o valoración de los objetos expuestos para referirse principalmente a los aspectos físicos de la vulnerabilidad. Más allá de expresar una posibilidad de daño físico, es crucial reconocer que los riesgos pueden ser inherentes, aparecen o existen dentro de sistemas sociales. Igualmente es importante considerar los contextos sociales en los cuales los riesgos ocu-rren; por consiguiente, la población no necesariamente comparte las mismas percepciones sobre el riesgo y sus causas subyacentes (pp. 18 - 19).

Bajo las anteriores consideraciones es consistente asumir la conceptualización sobre el riesgo que propone Olcina Cantos (2008), al definirlo como la plasmación (sic) territorial de una actuación humana poco acorde con los rasgos del medio donde tienen lugar o como una infracción que el hombre comete sobre el territorio por la implantación inadecuada de actividades o asentamientos. Dicha implantación de usos en el suelo no se ajusta, por desconocimiento o imprudencia, a las características físicas del medio. Reconociendo, por último, que existen diferentes enfoques para la definición del concepto riesgo «natural», según se otorgue prioridad al componente natural, social o territorial que forman parte de él.

A la luz de los anteriores conceptos y en el marco de la relación teórica que proponen, Herzer et al (2003) plantean una definición social de desastre a la que intentan "introducir elementos del mundo natural y social"; para introducir 
explícitamente el concepto de gestión del riesgo, al proponer que ésta "abarca, tanto las estrategias de atención en torno a la emergencia, la rehabilitación y la reconstrucción, como las políticas y acciones de mitigación, el diagnóstico, la preparación y la prevención de los desastres." (pp. 9 - 10)

De los conceptos de Olcina Cantos (2008) se puede extraer su proposición en el sentido de que el concepto riesgo natural define y determina la probabilidad de que un grupo social pueda verse afectado por un fenómeno natural de rango extraordinario que se desarrolle en el espacio geográfico donde se localiza dicha comunidad.

Concluye Olcina Cantos (2008) su línea de pensamiento con la propuesta de una "asociación" teórico - conceptual entre riesgo y territorio e invita a entenderla como un nuevo concepto, que refleja una nueva unidad de análisis territorial: la «región-riesgo» (...) un territorio de dimensiones conocidas afectado por uno o varios peligros naturales con incidencia sobre la población, los asentamientos y las actividades allí instaladas hasta el punto de suponer uno de los rasgos geográficamente más significativos de dicho espacio geográfico; en dirección a lo cual el riesgo latente en un territorio se convierte en un elemento de significación geográfica importantes en el análisis de dicho medio [y] llega a adquirir significación cultural y determina -condiciona- actuaciones de los seres humanos sobre el territorio orientadas a reducir o minimizar sus efectos (pp. 10 - 12).

Esta última reflexión de Olcina Cantos (2008) también lleva implícita una conceptualización sobre la gestión del riesgo que además tiene un hilo conductor con y en el ordenamiento del territorio, al definirlo como el proceso más eficaz para la reducción de la vulnerabilidad y exposición ante los peligros naturales. De este modo, si se acepta el concepto de región - riesgo propuesto por Olcina Cantos (2008), una de las funciones - objetivo del ordenamiento del territorio, en tanto territorio - riesgo, apuntará entonces a la reducción continua y progresiva de la proliferación de actuaciones humanas poco acordes con los rasgos naturales de su entorno.

En esa misma dirección, uniendo territorio y riesgo, se plantea un soporte conceptual para la gestión del riesgo que tiene como punto de partida asumir que dicha gestión es la estrategia para la reducción del riesgo (Ornés \& Chacón, 2009, pp. 3 - 6), la cual, a su vez, debe ser entendida como una de las funciones - objetivo del desarrollo sostenible, una de cuyas herramientas fundamentales será la planificación ambiental y territorial, cuyo propósito esencial es mejorar las condiciones de vida del individuo y la sociedad, reconociendo que las condiciones de vida de un individuo no solo están determinadas por su ubicación en la trama social sino también por su localización en la malla espacial, en la clásica e icónica interpretación del profesor Sergio Boisier (1979).

\section{MARCO METODOLÓGICO}

En las últimas décadas, en extensos territorios urbanos del mundo es evidente el aumento significativo del riesgo, no tanto por el incremento de las amenazas y/o de su peligrosidad, lo cual no ha sido consistentemente demostrado. Fundamentalmente, ello se ha originado en la proliferación de actuaciones e intervenciones humanas no acordes con los rasgos naturales de su entorno, en lo cual ocupa situación preponderante la acelerada y constante ocupación de territorios de elevada vulnerabilidad.

El análisis de la subcuenca del Arroyo León - Hondo, entendido como territorio-riesgo y en el contexto de la gestión del riesgo, se realizó bajo el enfoque metodológico FMPEIR ${ }^{5}$, el cual, según el PNUMA (2010), cubre y analiza las interrelaciones entre la sociedad humana y el medio ambiente haciendo énfasis en los servicios de los ecosistemas y su relación con el bienestar humano.

De acuerdo con el PNUMA (2010), en el enfoque metodológico FMPEIR, las Fuerzas Motrices o fuerzas indirectas se constituyen por los procesos fundamentales que dinamizan la sociedad e incluyen los cambios demográficos y los procesos económicos y sociales. Por su propia dinámica, estas fuerzas generan Presiones sobre el entorno que se expresan en cambio en el uso de la tierra, la extracción de recursos, las emisiones de contaminantes y desechos, y la modificación y el desplazamiento de organismos. El medio ambiente se ve alterado y sufre cambios en su Estado, originados en dichas presiones, cambios estos que se suman a los cambios naturales: el cambio climático, el agotamiento del ozono estratosférico, cambios en la biodiversidad y la contaminación o degradación del aire, el agua y los suelos, son los más percibidos. Ellos se evidencian en cambios en los bienes y servicios ambientales, como la disponibilidad de aire y agua limpios, de alimentos y de protección de la radiación ultravioleta. Estos cambios ambientales, unidos a los factores demográficos, sociales y materiales, se traducen, a su vez, en Impactos negativos sobre la salud, los activos materiales, las buenas relaciones sociales y la seguridad, los cuales son indicadores del bienestar de la sociedad. Ésta realiza intentos formales y no formales en dos direcciones: para adaptarse a los cambios de los bienes y servicios ambientales o para disminuir las presiones sobre el medio ambiente, son las que el PNUMA denomina Respuestas. El marco metodológico enunciado entiende que en la gestión integrada de la Subcuenca del Arroyo León - Hondo se integran el concepto de manejo del territorio-riesgo, la gestión del recurso hídrico y el ordenamiento territorial; lo anterior implicó una comprensión integrada ${ }^{6}$ de diversos factores que intervienen en la gestión de los recursos

5

5

6 
ARTE \& DISEÑO, ISSN: 1692-8555, Vol. $11 \mathrm{~N}^{\circ} 1$, Enero - Junio 2013 Gestión integrada de la subcuenca del arroyo León - Hondo en el occidente del suelo urbano del distrito de Barranquilla, Colombia, págs. 47-62

de la cuenca entre los cuales se contemplan, al menos: Los principales determinantes de la gestión de la cuenca (agua y territorio) y sus recursos naturales con los ecosistemas relacionados.

- La determinación de las prioridades en el uso del agua, buscando maximizar los beneficios globales y reducir los conflictos entre los que compiten y dependen de este recurso.

- El equilibrio de los intereses económicos, sociales y ambientales, tanto de los usuarios directos de la cuenca como de la sociedad en su conjunto.

- La cantidad y calidad del agua disponible y el tiempo de ocurrencia de fenómenos asociados a ésta.

- La integración de la gestión en el ámbito de las microcuencas, acuíferos y sistemas hídricos interconectados.

- La integración de la gestión de la demanda de agua con la gestión de la oferta.

\section{EL ÁREA OBJETIVO: SUBCUENCA DEL ARROYO LEÓN - HONDO LOCALIZACIÓN Y CARACTERÍSTICAS DEL ENTORNO}

La Subcuenca del Arroyo León - Hondo forma parte de la cuenca de la Ciénaga de Mallorquín, la cual cubre un territorio que abarca una superficie de 296.218 hectáreas y se encuentra ubicada al costado occidental del perímetro del suelo urbano de Barranquilla. Incluye el área de la ciénaga misma, que tiene una superficie de 857 hectáreas, y la ciénaga de Los Manatíes, con una extensión de 71 hectáreas $\left(\mathrm{CRA}^{7}, 2006\right.$, pp.113).

La superficie de la cuenca de la Ciénaga de Mallorquín está definida por los divorcios de los arroyos Santo Domingo y Hondo, en el costado occidental del Distrito de Barranquilla; en sectores del municipio de Puerto Colombia por la trayectoria del Arroyo León; en jurisdicción del municipio de Baranoa, por el divorcio de las aguas de los arroyos Jubilado, Simón y Megua; en el municipio de Tubará, por los divorcios de los arroyos Batuque, Malemba, Horno, San Luis, Don Juan y Granada; y en el municipio de Galapa en su totalidad, por los divorcios de los arroyos Mollo, Grande y Caña (Ver gráfico anterior).

En los 296,22 km2 del territorio de la Cuenca de Mallorquín tienen jurisdicción los municipios de Barranquilla (30,8 \% del área total), Galapa $(30,2 \%$ de la cuenca), Puerto Colombia (18,9\% del total), Tubará (Baranoa $(5,8 \%)$ y Soledad $(0,6 \%)$.

En el POMCA de la Ciénaga de Mallorquín se describe el relieve de la cuenca como definido por una planicie pequeña que se forma muy cerca de la ciénaga de Mallorquín y que se extiende con unos $5 \mathrm{Km}$. de ancho hasta Punta Roca sobre el costado occidental de la ciénaga. En esta pequeña planicie está incluida la ciénaga de Los Manatíes. Siguiendo hacia aguas arriba por el arroyo Grande, su principal afluente, el ancho de la planicie se reduce formando un valle estrecho entre dos pequeños promontorios; justo arriba del estrechamiento, se encuentra la confluencia de los arroyos Grande y Hondo (o León). (...) Luego el valle se abre para dar inicio a la gran planicie que domina el cuerpo central de la cuenca; el relieve es un ondulado suave que se va empinando a medida que se acerca a las divisorias de agua. En esta planicie discurren los afluentes principales del arroyo Grande: el arroyo Granada, que nace en las lomas de La Peronilla y Santa Rosa, junto a población de Tubará, y el arroyo Hondo $^{8}$, que nace en la Loma de La Sierra, entre las poblaciones de Galapa y Caracolí. Se considera que este modelado de planicie con ondulaciones suaves se extiende aproximadamente hasta la cota $100 \mathrm{msnm}$ y representa el $62 \%$ del área de la cuenca. El relieve quebrado sigue a continuación hasta las divisorias (...) y constituye el $38 \%$ restante (CRA et al, 2006, p. 91).

Como prácticamente la totalidad de los arroyos de Barranquilla y del departamento del Atlántico, el Arroyo León - Hondo es de origen pluvial, y según la descripción del POMCA ${ }^{9}$, el arroyo Hondo está conformado por el arroyo Caña, que nace en la loma de La Sierra y recibe los aportes del arroyo Santo Domingo. La confluencia de los arroyos Santo Domingo y Cañas forman el arroyo Hondo, el cual recibe todas las descargas del drenaje pluvial del sector de la ciudad dentro de la cuenca, así como las aguas residuales provenientes de la planta de tratamiento. A causa de estos aportes de la planta, el arroyo Hondo mantiene un caudal más o menos constante en la época de verano (CRA et al, 2006, p. 114).

De otra parte, las escorrentías urbanas que recibe la Cuenca de Mallorquín provienen en su totalidad del área urbana de Ba-rranquilla, de acuerdo con la descripción que realiza el mismo POMCA (CRA et al., 2006, pp. 115). Este drenaje recibe las escorrentías superficiales que se originan en los barrios del suroccidente y el occidente del suelo urbano de Barranquilla y conducen sus descargas hasta el arroyo Santo Domingo (nombre que recibe el cauce en el suelo urbano al atravesar un importante sector de la ciudad que lleva este nombre), el cual se denomina más adelante Arroyo Hondo y/o Arroyo León.

A partir de las características hidrográficas de la Cuenca de Mallorquín, se propone su división en las siguientes subcuencas (ver el gráfico siguiente): León - Hondo (9.933 ha.), Grande (6.449 ha.), San Luís - Granada (7.533 ha.), Lago del

Teniendo en cuenta que los nombres de Arroyo Hondo y Arroyo León son denominaciones que recibe el mismo cauce en distintos sectores de su recorrido en este trabajo se habla de la subcuenca del Arroyo León - Hondo y se utilizarán indistintamente cualquiera de los 
Cisne (1.680 ha.), Mar Sabanilla - Salgar (410 ha.) y Subcuenca Baja (3.617 ha.). (CRA et al 2006, p. 126 y 264)

\section{CARACTERIZACIÓN DE LA SUBCUENCA DEL ARROYO HONDO - LEÓN}

Con una subcuenca de $99,3 \mathrm{~km} 2$ a la que le da su nombre y una longitud de $15,7 \mathrm{~km}$ entre su paso por la Avenida Circunvalar de Barranquilla y su confluencia en el Arroyo Grande para desembocar en la Ciénaga de Mallorquín, es evidente la importancia del Arroyo León - Hondo en la Cuenca.

El Arroyo León - Hondo tiene, entre sus principales afluentes, también pluviales, los arroyos Blanco, Mellas, El Salao, Cañas y Santo Domingo. Este último se constituye en su principal afluente urbano, por lo cual algunos, sin evidencia alguna, lo consideran como el propio arroyo y no un afluente.

Tal como se puede apreciar en los planos generales de la Cuenca de Mallorquín en el gráfico 4, la cobertura del suelo que predomina al oriente de la Avenida Circunvalar de Barranquilla es la de usos urbanos, lo cual se convierte en la fuente de las principales y más fuertes presiones a que está sometido el territorio de la Subcuenca.

En el Plan de Ordenamiento y Manejo de la Cuenca Hidrográfica de la Ciénaga de Mallorquín -POMCA- (2006), se plantea que esta subcuenca es la que probablemente presenta mayor grado de contaminación, debido al efluente de la laguna de oxi-dación y a los lixiviados del basurero de Barranquilla, además del drenaje urbano y aguas servidas(pp. 350); y, resaltando la evidencia empírica, afirma que, sobre las laderas que marcan el borde y límite del nororiente de la Subcuenca también se da el proceso de expansión urbana de Barranquilla. El norte de esta subcuenca, al sur de la carrera 46, en los últimos años ha presentado un aumento significativo en cobertura urbana, $\mathrm{y}$ todo indica a que esta dinámica se mantendrá. Otra situación interesante es la que se observa al sur de la vía de La Cordialidad, entre el núcleo urbano de Galapa y Soledad, en una matriz de pastos en relieves planos y disectados, un cinturón de construcciones dispersas. Poco a poco este sector se irá poblando hasta convertirse seguramente en cobertura urbana. A lo largo de este sector y desde el norte al sur, atraviesan la cuenca un par de líneas eléctricas de alto voltaje.

\section{DINÁMICA Y FORMA DE OCUPACIÓN DEL TERRITORIO}

Como se puede derivar del gráfico siguiente, el área de la Subcuenca del Arroyo León - Hondo se caracteriza por una estructura de ocupación de sus suelos en la que el $63,3 \%$ de la superficie de la subcuenca se reparte entre una aparente cobertura arbórea $(29,6 \%)$ y pastos $(33,7 \%)$. En magnitud, a las superficies descritas sigue la cobertura en usos urbanos, que representa el 22,7 \% del área de la subcuenca, en la cual el mayor peso lo tiene el Suelo Urbano de Barranquilla.

Del análisis de la distribución de la cobertura de pastos en el mapa del gráfico anterior se evidencia que su distribución en la superficie ocupa las áreas menos pendientes del territorio objetivo, y se puede apreciar la coincidencia de esta cobertura con la presencia de la mayoría de los embalses (cuerpos de agua cerrados artificialmente) existentes.

\section{DINÁMICA DE POBLACIÓN}

La población de la Subcuenca en 2011 se estimó a partir de la información consignada en el POMCA de Mallorquín (CRA et al., 2006, p. 218 - 219), teniendo en cuenta los siguientes factores:

- La línea que separa las aguas de la Subcuenca del Arroyo Grande de las aguas de la Subcuenca del Arroyo León Hondo, atraviesa el municipio de Galapa dividiendo, tanto su Suelo Urbano como el resto del municipio y dejando aproximadamente el $40 \%$ de estos suelos en el área perteneciente a la Subcuenca del Arroyo León - Hondo ${ }^{10}$, mientras el $60 \%$ restante de la superficie del municipio se reparte entre las Subcuencas del Arroyo Grande y del Arroyo San Luis - Granada.

- El DANE ${ }^{11}$ calculó que la población total de Barranquilla llegaba a 1.193.952 habitantes y la población total de Galapa a 38.186 habitantes en 2011.

- Con estos criterios, se pudo asumir consistentemente que la población total de la Subcuenca en estudio en 2011 ascenderá a casi 414.000 personas, distribuidas de la siguiente manera en las jurisdicciones territoriales:

- La porción territorial de la Subcuenca que corresponde a Barranquilla y que representan el $33,4 \%$ de la población total de la ciudad estimada a 2011 por el DANE, alcanza unas 398.593 personas, aproximadamente.

- En la jurisdicción del municipio de Galapa, la subcuenca alberga al $40 \%$ de la población total proyectada por el DANE para el municipio; según esto, a 2011 habitan aproximadamente 15274 personas en esta parte.

A partir de los datos obtenidos del censo DANE $2005^{12}$, se estiman 83.379 viviendas pertenecientes a Barranquilla y 3.267 pertenecientes a Galapa que, en 2011, ocupaban la Subcuenca del Arroyo León - Hondo para un total de casi 86.650 viviendas en el área de estudio.

10

11

124 personas por vivienda para Barranquilla y 4,7 personas por vivienda para Galapa. 
ARTE \& DISEÑO, ISSN: 1692-8555, Vol. $11 \mathrm{~N}^{\circ} 1$, Enero - Junio 2013 Gestión integrada de la subcuenca del arroyo León - Hondo en el occidente del suelo urbano del distrito de Barranquilla, Colombia, págs. 47-62

Los volúmenes de aguas servidas que vierten estas viviendas en la subcuenca se pueden estimar asumiendo las dotaciones establecidas por el RAS ${ }^{13}$, así: 1.527,4 m3 provenientes de Galapa y 59.789 m3 de Barranquilla, lo cual significa que en la Subcuenca del Arroyo León - Hondo se estarían vertiendo aproximadamente $0,71 \mathrm{~m} 3 / \mathrm{seg}^{14}$.

\section{DINÁMICA DE LAS ACTIVIDADES}

De las seis subcuencas que configuran la Cuenca de la Ciénaga de Mallorquín, la del Arroyo León - Hondo, con el 33,5\% de la superficie total y el $88,5 \%$ de los habitantes de la cuen$\mathrm{ca}$, es la que genera las principales y mayores presiones sobre este territorio, puesto que cualitativa y cuantitativamente está influenciada por la dinámica urbana y suburbana de Barranquilla, las cuales se convierten en los factores que provocan los constantes y significativos cambios en el estado del medio am-biente de la Subcuenca. Las investigaciones de campo, las consultas de información secundaria, así como los talleres con la comunidad y actores principales de la cuenca, llevados a cabo durante la elaboración y formulación del POMCA de la Ciénaga de Mallorquín (CRA et al., 2006, p. 150 - 151), permitieron detectar tres factores - problema generadores de presiones directamente asociadas con la administración y gestión del territorio y del recurso hídrico en el área de la Subcuenca objeto de estudio: la contaminación de los cauces originada por residuos líquidos y sólidos; las escorrentías superficiales urbanas que se generan en Barranquilla; y el represamiento irregular concomitante con el aprovechamiento indebido de los cauces, todo ello, proveniente de actividades generadas por la dinámica urbana y suburbana de Barranquilla.

\section{INTERRELACIONES SOCIEDAD - MEDIO AMBIENTE EN EL ÁREA OBJETIVO. INTRODUCCIÓN: EL ENFOQUE METODOLÓGICO FMPEIR}

Como se anotó, el Plan de Gestión Integrada de la Subcuenca del Arroyo León - Hondo en el occidente del suelo urbano del Distrito de Barranquilla (Colombia) propone, como enfoque metodológico para el análisis, el denominado FMPEIR (Fuerzas Motrices - Presión - Estado - Impacto - Respuestas) por el PNUMA (2010), el cual apunta a cubrir y analizar las interrelaciones entre la sociedad humana y el medio ambiente haciendo énfasis en los servicios de los ecosistemas y su relación con el bienestar humano.

13

un estimativo conservador, 150 litros/habitante/día para sistemas de complejidad alta (Barranquilla) y 100 litros/habitante/dia para sistemas de complejidad baja (Gaapa)
En esa misma dirección, el PNUMA (2010, p. 31) precisa que el marco FMPEIR pretende reflejar los componentes clave de la compleja cadena causa-efecto que tiene lugar en el espacio y en el tiempo, y que caracteriza las interacciones entre sociedad y medio ambiente. Los cambios ambientales se ven inducidos por factores desencadenantes y están motivados por presiones, pero también se afectan los unos a los otros. Dichos cambios interactúan con factores demográficos, sociales y materiales en el momento de determinar el bienestar humano. Las respuestas incluyen medidas por parte de la sociedad para mitigar los cambios ambientales y adaptarse a ellos. Estos procesos tienen lugar en todas las escalas espaciales, desde la global hasta la local.

En síntesis, el enfoque en la perspectiva FMPEIR se constituye en un ejercicio de análisis en el cual se identifican las fuerzas motrices (factores sociales, económicos y políticos) que condicionan y producen un conjunto de presiones que, a su vez, operan sobre el ambiente (acciones antrópicas sobre los ecosistemas, extracción de recursos y/o la descarga de contaminantes), donde se incluyen también las dinámicas ecológicas de respuesta (resiliencia de los ecosistemas). Estas últimas determinan el estado del ambiente (situación de las áreas naturales, la calidad de suelos o de las aguas), lo cual es cuantificable y calificable por medio de evaluaciones ambientales integradas. Los efectos concretos de las presiones sobre el estado del ambiente son descritos, a su vez, por los impactos (por ejemplo: problemas sobre la salud humana o deterioro de la calidad de vida causado por baja calidad ambiental). Por último, el FMPEIR define las respuestas, que incorporan las acciones para enfrentar la situación ambiental (estado), para corregir o racionalizar las presiones que determinan y explican los impactos y para reparar o ajustar los impactos en sí mismos (más estricta normatividad ambiental, tecnologías reductoras de la contaminación, extender la educación ambiental).

En dirección a lo anterior, se sintetizan los elementos FMPEIR de los procesos que interactúan para explicar la condición socio-ambiental de la Subcuenca del Arroyo León - Hondo.

\section{FUERZAS MOTRICES}

La evidencia empírica ha demostrado que en la situación actual de la Subcuenca del Arroyo León - Hondo, concurren al menos las siguientes Fuerzas Motrices que están contribuyendo a su degradación ambiental, a la ocupación insostenible de su territorio, al deterioro progresivo de sus recursos hídricos y al modelo conflictivo de uso del suelo en su ámbito:

- Crecimiento urbano incontrolado de Barranquilla, tanto en sectores formales de estratos socioeconómicos altos en la franja nororiental de la subcuenca como en sectores marginales y formales de estratos socioeconómicos bajos 
en su borde Sur-oriental, los cuales conforman el borde suroccidental, occidental y noroccidental del suelo urbano de Barranquilla.

- Elevados índices de pobreza y, en algunos sectores, alto grado de miseria, en la franja suroriental y oriental de la subcuenca.

- Presencia de actividades económicas productivas: granjas agropecuarias de diverso propósito y tamaño, así como explotación legal e ilegal de canteras.

- Ubicación de actividades y macro-infraestructura para la disposición final de residuos líquidos (EDAR del barrio El Pueblo) y sólidos urbanos (Relleno sanitario "El Henequén") por parte de empresas y autoridades sanitarias de Barranquilla.

- Debilidad normativa y superposición de entidades para la regulación y el control ambiental y el ordenamiento ambiental y territorial, lo que contribuye a debilitar la capacidad y la gestión institucional en el ámbito de la subcuenca.

- Aislamiento, baja accesibilidad y débil conectividad con el sistema urbano de Barranquilla, lo que convierte a la Subcuenca en "territorio de nadie".

- Patrones incontrolados de consumo urbano existentes en la población de Barranquilla residente en el borde de la subcuenca.

\section{PRESIONES}

Las fuerzas motrices descritas generan diversos tipos de presiones, las cuales se evidencian, entre otras, en acciones antrópicas sobre los ecosistemas, en extracción de recursos y/o en la descarga de contaminantes en la Subcuenca del Arroyo León - Hondo:

- Obstrucción de cauces y rondas hidráulicas por la disposición de grandes volúmenes de residuos sólidos orgánicos e inorgánicos, construcción de represas o reservorios, desvíos y ocupación ilegal.

- Remoción de masas de tierra en las laderas, sobre todo en época de lluvias.

- Relleno ilegal de los cauces y cuerpos de agua para la expansión del suelo agrícola y pecuario y/o la construcción de infraestructura e instalaciones de diversa índole.

- Pavimentación e impermeabilización de extensas superficies urbanas.

- Práctica de quemas para cultivos, especialmente en zonas pendientes.

- Ocupación de laderas inestables y deleznables con infraestructura y construcciones de todo tipo, especialmente viviendas.

- Usos conflictivos del suelo en el territorio de la subcuenca.

- Deforestación generalizada, la cual se expresa en la eliminación del bosque primario y tiene entre sus consecuencias el desplazamiento y/ó la modificación de especies y la alteración del bioma.

- Explotación no siempre regulada y controlada de recursos mineros (calizas y arenas).

- Vertimiento de todo tipo de residuos sólidos y líquidos de origen doméstico, residuos industrial y de lixiviados sobre los cauces, los cuerpos de agua y sus rondas hidráulicas.

\section{ESTADO}

La situación de las áreas naturales, la calidad de suelos o de las aguas, en general, la condición ambiental de la Subcuenca del arroyo León - Hondo reflejan el estado del ambiente en el territorio, lo cual, como se anotó, se puede dimensionar cuantitativa y cualitativamente por medio de evaluaciones ambientales integradas $y$, aunque esto no está dentro del alcance de este trabajo, puede enunciarse en los siguientes aspectos:

- Incrementos del volumen, caudal y fuerza de las escorrentías provenientes del Suelo Urbano de Barranquilla y el consecuente aporte masivo puntual de las aguas por encima de los estimados para períodos largos de retorno.

- Reducción de la capacidad de los cauces por sedimentación, derrumbes u obstrucción de los cauces por acción antrópica

- Daños en la infraestructura productiva, económica, de comunicaciones y de servicios públicos domiciliarios.

- Incendios forestales, pérdida de capa vegetal con la consecuente desprotección del suelo y elevada posibilidad de erosión por escorrentías superficiales.

- Desertización y degradación progresiva de los suelos, asociadas a la lenta regeneración de la vegetación debido a prolongados períodos de verano y de estiaje, así como también asociadas al endurecimiento de los horizontes de los suelos de la parte plana, el cual se origina en cementaciones de carbonatos de calcio que impiden la profundización radicular, la adecuada aireación, la buena permeabilidad y el buen drenaje, todo lo cual desfavorece el adecuado comportamiento productivo. (POMCA de la Ciénaga de Mallorquín, CRA et al, 2005)

- En las áreas colinadas de la Subcuenca Alta, se presentan elevados niveles de salinidad en los suelos asociados a la concurrencia de bajas precipitaciones y alta evapotranspiración, lo cual facilita la permanencia de sales y sodio en el perfil del suelo, las cuales son lavadas por las aguas de escorrentía, con el consecuente aumento de la salinidad de los suelos en las zonas planas y bajas. (CRA et al., 2005)

- Pérdida de la capacidad productiva intrínseca de los suelos en la parte baja por déficit en la oferta de agua, la cual es retenida incontrolada e ilegalmente aguas arriba de la subcuenca. 


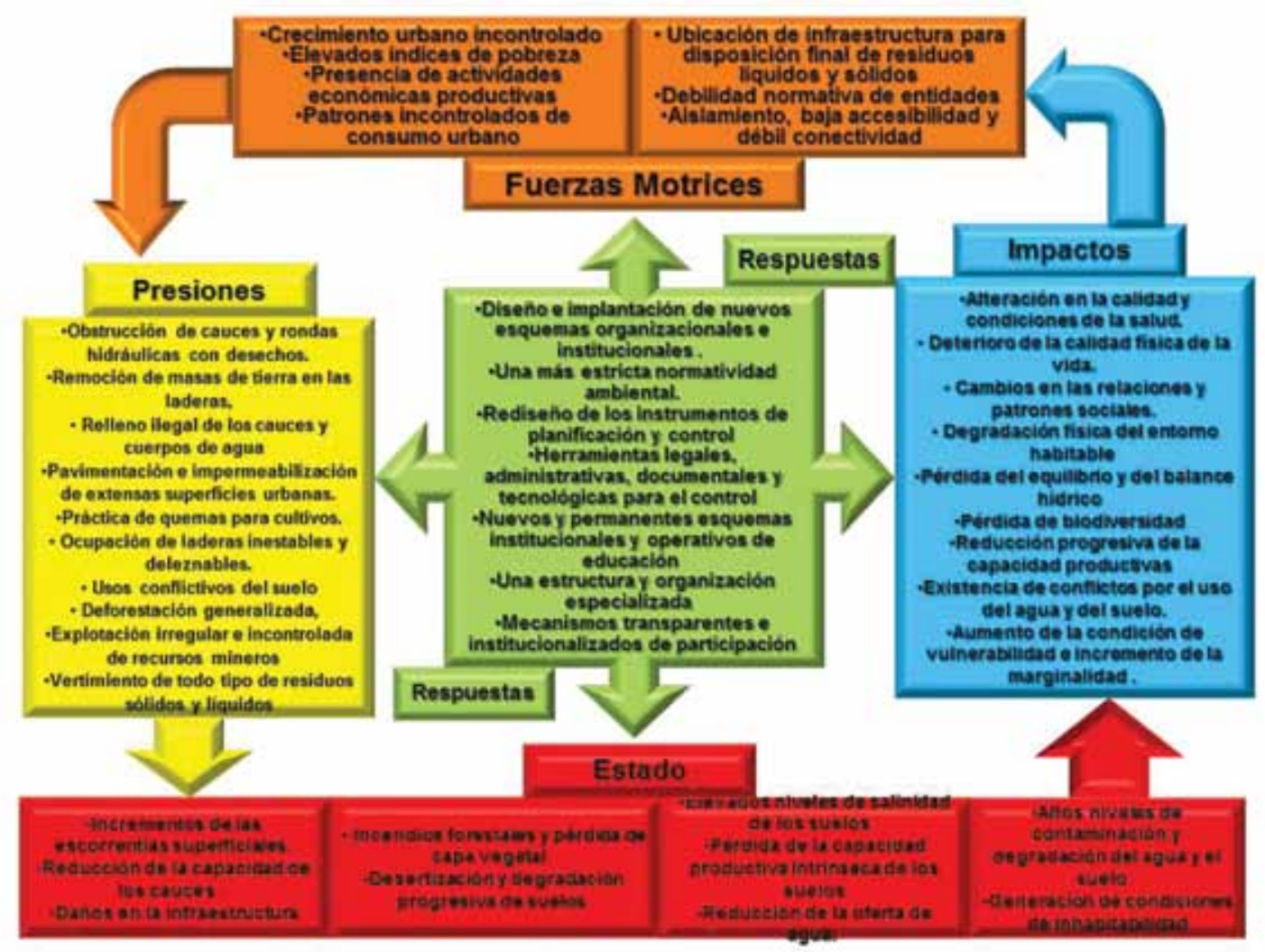

Gráfico 1. Factores - problema generadores de presiones cauce del Arroyo León - Hondo

Fuente: Fotografias captadas durante recorridos realizados en noviembre de 2010 (Izq.) y video realizado por Estudiantes de la Universidad del Norte de Barranquilla, curso de Legislación Ambiental (junio de 2008). (Bajado de http://www.youtube.com/watch?v=Hr77_bct_AU) (Der.)

- Reducción de la oferta de agua en la medida que se va llegando a las áreas más bajas de la subcuenca.

- Altos niveles de contaminación y degradación del agua y el suelo por presencia de patógenos, material no biodegradable, sólidos en suspensión, compuestos inorgánicos y metales pesados.

- Generación de condiciones de inhabitabilidad en ciertos sectores.

\section{IMPACTOS}

Como se ha anotado, los efectos concretos de las presiones sobre el estado del ambiente son explicados por los impactos, los cuales se reflejan en situaciones - problemas causadas por la baja calidad y el deterioro ambiental, tales como:

- Alteración en la calidad y condiciones de la salud pública, individual y colectiva.

- Deterioro de la calidad física de la vida.
- Cambios en las relaciones y patrones sociales de los individuos y sus colectividades.

- Degradación física del entorno habitable, con la consecuente pérdida del bienestar humano individual y colectivo.

- Aumento de la condición de vulnerabilidad e incremento en el "círculo vicioso" de la marginalidad ecológica y urbana.

- Pérdida del equilibrio y del balance hídrico en la subcuenca por retención en reservorios, represas y desviación de los cauces de agua.

- Pérdida de biodiversidad por la desaparición de bosques, ruptura de la conectividad entre ecosistemas, alteración de las estructuras ecológicas territoriales, y desaparición, transformación ó desplazamiento de especies.

- Reducción progresiva de la capacidad y posibilidades productivas de la Subcuenca y, por ende, de las capacidades de satisfacción de las necesidades materiales de los pobladores.

- Existencia de conflictos por el uso del agua y del suelo. 


\section{RESPUESTAS}

Las respuestas se refieren a las decisiones y acciones que toma la sociedad para mitigar o enfrentar los cambios ambientales, adaptarse a ellos, corregir las presiones y/o reparar los impactos, en dirección a lo cual se plantea el presente Plan de Gestión Integrada de la Subcuenca del Arroyo León - Hondo, el cual apuntará a definir lineamientos y propuestas que permitan implementar, al menos:

- Regularizar los vertimientos de aguas servidas de las diferentes actividades para incorporarlas o conectarlas a las redes de alcantarillado sanitario $y$, por éstas, al sistema de tratamiento de la EDAR del barrio El Pueblo.

- Optimizar el sistema de tratamiento de la EDAR, para garantizar que las aguas de su efluente cumpla sobradamente con los parámetros de calidad exigidos por la normatividad internacional (más exigente que las colombianas).

- Establecer estrictos mecanismos para regular y controlar los embalses y desvíos de las aguas de la cuenca.

- Revisar y ajustar el proceso de postclausura del relleno sanitario El Henequén, con el fin de aplicar correctivos técnicos que controlen o eviten el vertimiento de sus lixiviados en los cauces de la Subcuenca.

- Diseñar, implementar y poner en marcha programas de repoblamiento de especies vegetales y animales.

- Diseño e implantación de nuevos esquemas organizacionales e institucionales que maximicen la coordinación, definan responsabilidades, precisen competencias y establezcan compromisos reales y factibles de acción intra e interinstitucional, tanto en el ámbito municipal como en el ámbito metropolitano.

- Una más estricta normatividad ambiental, que incorpore mecanismos de prevención, control, seguimiento, compensación y sanción frente al uso indiscriminado e irregular del recurso hídrico y del territorio.

- Rediseño de los instrumentos de planificación, control, regulación y ejecución estratégica del ordenamiento del territorio y del recurso hídrico

- Disponibilidad de herramientas legales, administrativas, documentales y tecnológicas para el control y la reducción de la contaminación y degradación ambiental, especialmente la hídrica y edáfica en la subcuenca.

- Implementación de permanentes esquemas institucionales y operativos que induzcan procesos consistentes de educación para la prevención, el control y la conservación ambiental.

- Una estructura y organización especializada para el control y la sanción que aplique eficazmente la normatividad vigente.

- Diseño y puesta en marcha de mecanismos transparentes e institucionalizados de participación comunitaria en la gestión ambiental y del recurso hídrico, entendiendo que en la medida en que existan procesos de apropiación del colectivo social sobre los recursos de la subcuenca se ampliará el espectro de pertenencia y, consecuentemente, de protección del mismo.

\section{FACTORES - PROBLEMA EN LA SUBCUENCA DEL ARROYO LEÓN - HONDO CARACTERÍSTICAS DEL PROBLEMA}

Algunos autores afirman que en la superficie terrestre existen territorios de riesgo e incluso puede hablarse de paisajes de riesgo, esto es, espacios cuya característica geográfica principal es la inadecuada ocupación de un espacio que soporta frecuentes eventos naturales de rango extraordinario (Olcina Cantos, 2008, p. 10).

El mismo autor asevera que con mucha frecuencia estos territorios o paisajes de riesgo se encuentran localizados en áreas urbanas de los países menos desarrollados. En esta perspectiva, es consistente pensar que si bien hasta el presente el territorio de la Subcuenca del Arroyo León -Hondo, objeto de este estudio, no ha soportado frecuentes eventos naturales de rango extraordinario, la concurrencia de numerosas Presiones, aunque aún puedan catalogarse de "pequeñas" como las descritas con anterioridad, lo convierte en un territorio o paisaje de riesgo.

De este modo, con las Presiones que caracterizan los factores - problema de la Subcuenca del Arroyo León - Hondo se han traducido en cambios en el Estado del medio ambiente del área, dichos cambios ambientales se pueden sintetizar de la siguiente manera:

- Deterioro y contaminación de los cauces (aguas y suelos), lo cual se evidencia en:

- Disposición de residuos sólidos orgánicos e inorgánicos.

- Vertimiento de aguas residuales domésticas sin tratar.

- Descarga del efluente de la EDAR del barrio El Pueblo

- Filtración de los lixiviados provenientes del relleno sanitario El Henequén de Barranquilla.

- Configuración de un modelo de ocupación desequilibrante del territorio que soporta la Subcuenca del Arroyo León Hondo, que se manifiesta en:

- Ocupación formal e informal, con grandes intervenciones urbanísticas, de las laderas deleznables del borde oriental de la subcuenca.

- Deslizamientos de grandes masas de suelo, infraestructura y construcciones.

- Generación de ingentes volúmenes de escombros y de material de arrastre.

- Incremento de las escorrentías superficiales por la impermeabilización de extensas superficies, lo cual disminuye el aporte gradual a la cuenca y genera grandes caudales que llegan de manera puntual a los cauces de los arroyos de la Subcuenca y que desestabilizan aún más las laderas. 
ARTE \& DISEÑO, ISSN: 1692-8555, Vol. $11 \mathrm{~N}^{\circ} 1$, Enero - Junio 2013 Gestión integrada de la subcuenca del arroyo Leon - Hondo en el occidente del suelo urbano del distrito de Barranquilla, Colombia, págs. 47-62

- Desestabilización de orillas de los arroyos por la erosión causada por las grandes avenidas "puntuales" de los arroyos urbanos que desembocan en la subcuenca.

- Conflictos por el uso del agua de la subcuenca, lo cual se aprecia en factores como:

- Ocupación irregular de las rondas hidráulicas.

- Represamiento irregular y antitécnico de las aguas de los arroyos.

- Aprovechamiento ilegal de las aguas.

- Vertimientos fuera de norma.

- Pérdida de la biodiversidad y deterioro progresivo de los bienes y servicios ambientales, con el consecuente desequilibrio de la estructura ambiental de la subcuenca, que se evidencia en:

- Explotación indebida y la degradación acelerada de los recursos naturales.

- Disminución de la capacidad generadora de agua de las cabeceras de los afluentes.

- Alteración de los procesos ecológicos esenciales.

- Pérdida de la biodiversidad y la disponibilidad de servicios ambientales.

- Reducción cualitativa y cuantitativa de la oferta ambiental.

- Degradación de los suelos como consecuencia de las actividades de quema y deforestación irracional con la consecuente erosión, compactación y lixiviación de los mismos.

- Limitado control institucional sobre el territorio objetivo de la subcuenca, lo cual se manifiesta en:

- Jurisdicción de varios municipios en el territorio de la subcuenca.

- Superposición de entidades ambientales del orden local (DAMAB), Departamental (CRA) y Nacional (MAVDT) en el ámbito de la subcuenca.

\section{EL RIESGO EN EL ÁREA OBJETIVO CONCEPTO DEL RIESGO}

En tanto se entiende que la subcuenca del Arroyo León - Hondo es un concepto específico de comprensión de la dimensión de los atributos territoriales, la gestión integrada de la misma incorpora la gestión del riesgo a la gestión del recurso hídrico.

En la Estrategia Internacional para la Reducción de Desastres, EIRD, la ONU (ONU - EIRD, 2004, pp. 4 - 5) afirma que cada vez aumenta el número de sectores profesionales y gobiernos que coinciden en señalar que distintos segmentos de la población pueden verse expuestos a mayores riesgos relativos debido a desigualdades sociales o económicas que tornan más vulnerables sus condiciones de vida y que en razón a ello, la reducción de desastres se relaciona de manera creciente con los esfuerzos por alcanzar el desarrollo sostenible.

Concluye la $\mathrm{ONU}$ esta afirmación, anotando consistentemente que las relaciones entre la acción humana, la gestión ambiental, el cambio climático y el riesgo de desastres se están tornando cada vez más decisivas.
El punto de partida de la gestión del riesgo, en tanto concepto creador y generador de escenarios anticipativos y preventivos, es la pregunta sobre si la sociedad moderna $y$, en ella, los segmentos más pobres, los marginados ecológicos y urbanos (Santiago, 1984, pp. 53 - 71), pueden continuar realizando la valoración de sus activos (ambientales, sociales, productivos y materiales, tanto individuales como colectivos) solamente después de perderlos a causa de un desastre.

En los años recientes, la cada vez mayor frecuencia y gravedad de los desastres de origen natural, las poblaciones locales afectadas -a través de sus autoridades, técnicos, gremios de la producción y el comercio, organismos públicos, sector educativo y dirigentes han ido aceptando la necesidad de consolidar los esfuerzos para minimizar el costo social, económico y ambiental del impacto de los fenómenos originados por el desencadenamiento de las amenazas naturales.

De allí surge la necesidad de diseñar, formular, implementar, ejecutar y aplicar estrategias de anticipación, prevención y protección tendientes a evitar el riesgo para las vidas y proteger bienes y recursos antes de que se pierdan.

A partir de esa necesidad de crear escenarios anticipativos y preventivos surge el concepto de reducción del riesgo o gestión del riesgo (de desastres), el cual enfatiza y prioriza los factores de vulnerabilidad y riesgo, desarrollado como un enfoque más holístico dentro del proceso de planificación y ordenamiento del territorio.

La reflexión de la ONU en la EIRD, (ONU - EIRD, 2004, p. 8) es consistente con lo anterior, según ésta en el fondo, se ha ido reconociendo de forma creciente que el riesgo de desastres guarda estrecha relación con los problemas ambientales y con cuestiones no resueltas que son fundamentales para el desarrollo sostenible. (...) Es preciso aplicar políticas y medidas que tengan el doble objetivo de lograr que las sociedades adquieran la resiliencia requerida ante las amenazas naturales y asegurar que los esfuerzos que realizan por alcanzar el desarrollo no aumenten su vulnerabilidad a dichas amenazas.

Herzer et al (2003) plantean la comprensión del desastre como un proceso, el cual se capta en la creación de las condiciones de riesgo a través del tiempo, como el resultado de la interacción entre determinados eventos desencadenantes (amenazas) y las vulnerabilidades de la sociedad. En tal sentido, la probabilidad de que un evento desencadenante se convierta en desastre depende de la vulnerabilidad de la sociedad o de ciertos grupos sociales. Bajo este enfoque, el desastre es un continuo que incluye la generación de las condiciones de riesgo, la emergencia, y sus posteriores efectos sobre el territorio, la economía, la sociedad y la política.

De acuerdo con los expertos, el análisis de riesgo gira alrededor de tres conceptos que configuran el riesgo peligro (peligrosidad), vulnerabilidad y exposición, cada uno de los cuales está relacionado respectivamente con los tres elementos esenciales 
del espacio geográfico: la naturaleza, el hombre y el territorio. Esta relación se establece fundamentalmente, de la siguiente manera:

- El peligro (amenaza) debe ser entendido como la existencia real o potencial de un fenómeno o proceso de carácter natural o antrópico que puede originar daños a una comunidad, a sus actividades 0 al propio medio ambiente.

- La vulnerabilidad es la predisposición o posibilidad de pérdida potencial de un bien expuesto que debe entenderse en sus varias dimensiones (vulnerabilidad humana, vulnerabilidad estructural, vulnerabilidad económica y/o vulnerabilidad ecológica) de acuerdo con el tipo de riesgo que se esté midiendo. En este caso, el bien vulnerable por excelencia $y$, por ende, fundamental y prioritario, es la vida humana, por eso el grado de riesgo más elevado se considera cuando está en peligro la sobrevivencia de las personas.

- La exposición es la disposición -ubicación- sobre el territorio de un conjunto de bienes y/o personas, los cuales pueden ser dañados por un peligro natural o antrópico.

A la luz de la interpretación de Herzer et al. (2003, p. 5), se puede resumir que las características del riesgo serían: es una condición latente o potencial y su grado o intensidad depende de la intensidad que pueda alcanzar el evento peligroso o la magnitud posible de la amenaza, así como del nivel de vulnerabilidad que exista entre la población y el territorio. En esa dirección, las mismas Herzer et al. (2003) entienden que el riesgo es la probabilidad de ocurrencia de un desastre. $Y$ concluyen para que exista un riesgo, debe haber tanto elementos detonadores (sean de orden natural, socio-natural, antrópico y/o tecnológico), como una población vulnerable a sus impactos. Los desastres ocurren cuando no se conoce o no se actúa adecuadamente frente a los riesgos a los que estamos expuestos.

Así mismo, el DNP de Colombia (2005, p. 15), la gestión del riesgo se puede considerar parte o una dimensión de la gestión ambiental en tanto implica la revisión de la relación entre la sociedad y el medio ambiente y conduce a un modelo de intervención en el que se favorezcan ciertos usos del territorio y de sus recursos, al mismo tiempo que restringe otros, y concluye el DNP (2005), sintetizando que la gestión del riesgo comprende las actividades de identificación, monitoreo, prevención, mitigación y transferencia del riesgo, y de preparación para la atención, rehabilitación y reconstrucción frente a emergencias y desastres.

\section{GESTIÓN INTEGRADA DE LA SUBCUENCA DEL ARROYO LEÓN - HONDO. LAS RESPUESTAS DENTRO DEL ENFOQUE FMPEIR: MARCO DE AC- CIÓN DEL PLAN DE GESTIÓN INTEGRADA DE LA SUBCUENCA DEL ARROYO LEÓN -HONDO}

- Adaptación a los cambios en los servicios ambientales. Se considera que las respuestas que pretenden mitigar, adaptarse ó enfrentar los cambios ambientales dentro del Plan de Gestión Integrada de la Subcuenca del Arroyo León - Hondo apuntará a la puesta en marcha de acciones tendientes a:

- Eliminar progresivamente los vertimientos de aguas servidas sobre los cauces de la Subcuenca, en especial del propio Arroyo León - Hondo.

- Mejorar tecnológicamente la eficiencia y eficacia de la planta de tratamiento de la EDAR.

- Controlar el tamaño, localización y uso de los embalses y regularizar los desvíos de aguas de los arroyos que conforman la Subcuenca.

- Controlar el vertimiento de los lixiviados del relleno sanitario El Henequén.

- Rehabilitar y restaurar el bioma la subcuenca.

- Reducción de las presiones sobre el medio ambiente. Las respuestas que apuntan a reducir las presiones sobre el medio ambiente que se proponen dentro de la gestión integrada de la subcuenca del Arroyo León - Hondo considera fundamentalmente acciones de tipo institucional y normativo, partiendo de la premisa de que uno de los aspectos más críticos es la desinstitucionalización:

- Configuración de un nuevo ordenamiento institucional que cree verdaderos sistemas de coordinación; redefina el modelo de asignación de responsabilidad; establezca claras competencias; y acuerde compromisos de acción intra e interinstitucional, tanto en el ámbito municipal como en el ámbito metropolitano y departamental.

- Diseño, implementación y aplicación de una normatividad ambiental más estricta, en la cual se asegure la optimización de los procesos de prevención, control, seguimiento, compensación y sanción para combatir el uso irregular de los recursos de la Subcuenca.

- Rediseño y puesta en marcha de los necesarios instrumentos y mecanismos de planificación, control, regulación y ejecución estratégica del ordenamiento del territorio y del recurso hídrico.

- Instrumentar legal, administrativa, documental y tecnológicamente el control de las fuentes de contaminación y degradación ambiental en la Subcuenca.

- Diseño, implementación e institucionalización de un modelo interactivo y permanente que genere un proceso consistente de educación en materia de prevención, ordenamiento, control y conservación ambiental.

- Conformación de una estructura organizacional especializada para la aplicación de la normatividad vigente.

- Implementación e institucionalización de mecanismos de participación comunitaria en la gestión ambiental y del recurso hídrico, con el fin de expandir los procesos 
ARTE \& DISEÑO, ISSN: 1692-8555, Vol. $11 \mathrm{~N}^{\circ} 1$, Enero - Junio 2013 Gestión integrada de la subcuenca del arroyo Leon - Hondo en el occidente del suelo urbano del distrito de Barranquilla, Colombia, págs. 47-62

de apropiación del colectivo social, ampliar el sentido de pertenencia y generar acciones colectivas de protección sobre los recursos de la Subcuenca.

El gráfico muestra una visión holística de la gestión integrada de la Subcuenca del Arroyo León - Hondo y de las variables que deben integrarlo.

\section{CONCLUSIONES}

Herzer y Gurevich (2002), reconocen que los procesos de conformación del riesgo, la degradación y los desastres serán siempre objeto de intereses controvertidos. Esto es así porque dichos procesos se constituyen a partir de los encuentros y desencuentros de múltiples actores sociales y de racionalidades, intereses y lógicas diversas. En este sentido, las definiciones que se hagan de estos conceptos tendrán este mismo conjunto de limitaciones o condicionamientos $y$, al no ser neutras, suponen implícita o explícitamente la elección de una determinada escala de valores.

La anterior perspectiva sirve de elemento axial para proponer las conclusiones obtenidas en el Plan de Gestión Integrada de la Subcuenca del Arroyo León - Hondo en el occidente del suelo urbano del Distrito de Barranquilla (Colombia), así:

- La gestión integral del recurso hídrico necesariamente pasa por la gestión integral de las cuencas.

- Independientemente de su pequeña escala, la formulación de la propuesta de Plan de Gestión Integrada de la subcuenca del Arroyo León - Hondo, contiene todos los elementos que la harían expansible a ámbitos mayores.

- La gestión de la subcuenca debe ser concebida, entendida, desarrollada y ejecutada como un proceso integral, puesto que incorpora gestión ambiental, integra la gestión del recurso hídrico, involucra la gestión del ordenamiento territorial, contempla la gestión del riesgo y está indisolublemente asociada con la gestión de partes interesadas (actores).

- Para el logro de una exitosa gestión integral de la subcuenca (ó de las cuencas, en general) es necesario que el ordenamiento jurídico y la normatividad colombianas determinen institucional y legalmente las prioridades en los usos del agua, más allá de la simple enumeración que incluye el artículo $9^{\circ}$ del Decreto 3930 de 2010 (MAVDT, 2010) .

- Los Planes de Gestión Integrada de Subcuencas deben ser documentos oficialmente adoptados y aplicados con fuerza normativa en el que se indique cómo se coordinarán las iniciativas de todas las partes interesadas (tanto públicas como privadas), y que especifique las reglas y normas que habrán de implementarse en la cuenca. La declaración debe ser de fácil acceso para todas las partes interesadas y debe constar en formatos que todos puedan comprender.
- Finalmente, es necesario concluir que la gestión del riesgo se considera como una indisoluble parte de la Gestión Integrada de la Subcuenca del Arroyo León - Hondo porque entiende que la planificación del desarrollo debe tener presentes las siguientes reflexiones (PNUD, 2004):

- Las decisiones que se tomen en el presente pueden constituirse en riesgo de desastres en el futuro.

- Los movimientos y desplazamientos de población están cambiando el entorno de los riesgos de desastres.

- Los procesos de desarrollo modifican continuamente las amenazas naturales.

- La vida cotidiana se compone de los riesgos cotidianos, por lo cual la exposición cotidiana al peligro puede erosionar el potencial de desarrollo de la población hasta el punto de incrementar su vulnerabilidad futura.

- Los riesgos acumulados pueden desembocar en grandes desastres ante pequeños (o grandes) eventos.

- Los grandes desastres, con frecuencia, están "hechos" a partir de la "sumatoria" de pequeños desastres.

- La incorporación del riesgo en la planificación del desarrollo [territorial] significa tener en cuenta grandes y pequeños peligros.

\section{REFERENCIAS BIBLIOGRÁFICAS}

Boisier, S. (1979). La planificación del desarrollo regional en América Latina. Santiago de Chile: ILPES.

Cardona, 0. D. (1993). Manejo ambiental y prevención de desastres: dos temas asociados privado. En A. Maskrey, Los desastres no son naturales.

COMISIÓN ECONÓMICA PARA LA AMÉRICA LATINA (CEPAL). (s.f.). Obtenido de www.cepal.cl/publicaciones

Corporación Autónoma Regional del Atlántico. (2006). Plan de ordenamiento y manejo de la cuenca hidrográfica de la Ciénaga de Mallorquín (POMCA). Barranquilla: CRA.

DEPARTAMENTO ADMINISTRATIVO NACIONAL DE ESTADÍSTICA (DANE). (20 de Mayo de 2011). Estimaciones de población 1985 - 2005 y Proyeciones de población 2005 - 2020. Obtenido de www.dane.gov.co.

DEPARTAMENTO NACIONAL DE PLANEACIÓN (DNP). (2005). Guía para orientar las acciones e inversiones en gestión local del riesgo a nivel municipal. Bogotá, DC.: DNP.

GLOBAL WATER PARTNERSHIP (GWP); INTERNATIONAL NETWORK OF BASIN ORGANIZATIONS (INBO). (2009). Manual para la Gestión Integrada de Recursos Hídricos en Cuencas. Londres: GWP \& INBO.

Herzer, H., \& Gurevich, R. (julio - diciembre de 1996). Construyendo el riesgo ambiental en la ciudad. Desastres y Sociedad(7).

Herzer, H., Celis, A., Bartolomé, M., Rodríguez, C., \& Caputo, G. (2002). Convivir con el riesgo o la gestión del riesgo. Obtenido de La Red: http://www.cesam.org.ar/que.htm 
Herzer, H., Celis, A., Bartolomé, M., Rodríguez, C., \& Caputo, G. (2003). El manejo de cuencasmy su impacto en áreas urbanas: el caso de la llanura pampeana argentina. III Congreso Latinoamericano de manejo de cuencas hidrográficas. Arequipa,Perú: INRENA \& FAO.

MINISTERIO DE AMBIENTE, VIVIENDA Y DESARROLLO TERRITORIAL (MAVDT). (25 de 0ctubre de 2010). Decreto 3930. Por el cual se reglamenta parcialmente el Título I de la Ley $9^{\mathrm{a}}$ de 1979, así como el Capítulo II del Título VI -Parte III- Libro II del Decreto-ley 2811 de 1974 en cuanto a usos del agua y residuos líquidos y se dictan otras disposiciones. Bogotá, DC.: Diario Oficial.

Olcina, J. (2008). Cambios en la consideración territorial, conceptual y de método de los riesgos naturales. $X$ Coloquio Internacional de Geocrítica: Diez años de cambios en el mundo, en la geografía y en la Ciencias Sociales 1999 2008. Barcelona: Universidad de Barcelona.

ORGANIZACIÓN DE LAS NACIONES UNIDAS (ONU). (2004). Vivir con el riesgo: Informe mundial sobre iniciativas para la reducción de desastres. Ginebra: ONU \& EIRD.

ORGANIZACIÓN DE LAS NACIONES UNIDAS (ONU). (2004). Vivir con riesgo: Informe mundial sobre iniciativas para la reducción de desastres. Obtenido de www.eird.org: http://www. eird.org/vivir-con-el-riesgo/index2.htm

Ornés, S., \& Chacón, R. M. (2009). La gestión del riesgo ambiental como eje de la planificación urbana e indicador de desarrollo local. 1.

PROGRAMA DE LAS NACIONES UNIDAS PARA EL MEDIO AMBIENTE (PNUMA). (2004). Reducing disaster risk: A challenge for development - A global report. Obtenido de www.undp. org: http://www.eird.org/vivir-con-el-riesgo/index2.htm

PROGRAMA DE LAS NACIONES UNIDAS PARA EL MEDIO AMBIENTE (PNUMA). (2010). Perspectivas delmedio ambiente. América Latina y el caribe. GEO ALC 3. Panamá: PNUMA.

Santiago, F. (1984). Marginalidad Urbana: Dos estudios de caso. Barranquilla. (Tesis de Maestría, UNIANDES)

Solanes, M., \& Jouravlev, A. (2005). Integrando economía, legislación y administración en la gestión del agua y sus servicios en américa Latina y el caribe. Santiago de Chile: CEPAL.

\section{SITIOS DE INTERNET}

www.cepal.cl/publicaciones

www.eird.org/vivir-con-el-riesgo

www.ub.es/geocrit

www.desenredando.org

www.edafologia.ugr.es

www.dane.gov.co

http://www.aaa.com.co

http://www.panda.org 